\title{
BMJ Open Does androgen deprivation impact associations between cognition and strength, fitness and function in community-dwelling men with prostate cancer? A cross-sectional study
}

\author{
Niamh L Mundell (1) , ${ }^{1}$ Patrick J Owen (D) , ${ }^{1}$ Jack Dalla Via (1) , ${ }^{1,2}$ \\ Helen Macpherson (D) , Robin M Daly (D) , ${ }^{1}$ Steve F Fraser (iD ${ }^{1}$
}

To cite: Mundell NL, Owen PJ, Dalla Via J, et al. Does androgen deprivation impact associations between cognition and strength, fitness and function in community-dwelling men with prostate cancer? A cross-sectional study. BMJ Open 2021;11:e058478. doi:10.1136/ bmjopen-2021-058478

- Prepublication history and additional supplemental materia for this paper are available online. To view these files, please visit the journal online (http://dx.doi.org/10.1136/ bmjopen-2021-058478)

Received 18 0ctober 2021 Accepted 02 December 2021

Check for updates

(c) Author(s) (or their employer(s)) 2021. Re-use permitted under CC BY-NC. No commercial re-use. See rights and permissions. Published by BMJ.

${ }^{1}$ Institute for Physical Activity and Nutrition (IPAN), School of Exercise and Nutrition Sciences, Deakin University, Burwood, Victoria, Australia

${ }^{2}$ Institute for Nutrition Research, School of Medical and Health

Sciences, Edith Cowan

University, Perth, Western

Australia, Australia

Correspondence to

Niamh L Mundell;

niamh.mundell@deakin.edu.au

\section{ABSTRACT}

Objectives We investigated whether there were

differences in associations between cognition with muscle strength, fitness and function in men with prostate cancer (PCa) treated with, and without androgen deprivation therapy (ADT) and non-PCa controls. A secondary aim was to compare differences in the prevalence of cognitive impairment.

Design This cross-sectional study compared 70 ADTtreated men with PCa aged $50-85$ years to non-ADTtreated men $(n=52)$ and non-PCa controls $(n=70)$.

Setting University clinical exercise laboratory.

Interventions Nil.

Primary and secondary outcome

measures Standardised assessments were conducted for cognition (learning, memory, attention, processing speed and executive function), muscle strength (grip strength and leg press), fitness ( $400 \mathrm{~m}$ walk), gait speed (4 $\mathrm{m}$ walk) and dual-tasking mobility (timed-up-and-go with a cognitive task).

Results ADT-treated men showed stronger associations between fitness and executive function and task switching relative to controls (both: $p \leq 0.03$ ). For both PCa groups (independent of ADT use), poorer dual-task mobility was more strongly associated with decreased psychomotor attention (both: $p \leq 0.027$ ) and global cognitive function (both: $p \leq 0.031$ ) compared with non-PCa controls. The overall prevalence of cognitive impairment was low $(4 \%-$ $13 \%$ ) and did not differ between the groups

Conclusions The presence of PCa, with or without ADT treatment, did not increase the risk of cognitive impairment relative to non-PCa controls, yet did alter the associations between physical fitness and some measures of functional performance with certain cognitive domains. This highlights the importance of men with PCa maintaining fitness and functional capacity to optimise cognitive health.

Trial registration number This study was registered with the Australian and New Zealand Clinical Trials Registry (ACTRN12614000317695).

\section{INTRODUCTION}

Prostate cancer (PCa) has the second highest worldwide incidence of all male cancers $(14 \%$
Strengths and limitations of this study

- This study was strengthened by the use of a comprehensive battery of cognitive tests and a range of physical outcomes that related to strength, fitness and function.

- A further strength was that we followed recent guidelines from the International Cancer Cognition Task Force with regards to defining cognitive impairment

- A limitation is that this study was a nested crosssectional substudy within a larger randomised controlled trial, including exercise training and nutritional supplements for androgen deprivation therapy (ADT)-treated men; hence, men treated with ADT with a penchant for exercise may have been more likely to participate.

- Another limitation is that men on ADT recruited to the study had all been treated for a minimum of 3 months; thus, we are unable to draw any conclusions in men who have recently commenced ADT (ie, $<3$ months).

- Finally, given the cross-sectional design, causality cannot be established between fitness, physical function and certain cognitive domains with ADT.

in 2018), yet a high relative survival rate $(8 \%$ of cancer-related deaths). ${ }^{1}$ Androgen deprivation therapy $(\mathrm{ADT})$ is a mainstay in the treatment of appropriately selected men with metastatic and non-metastatic PCa. ${ }^{2}$ ADT is associated with numerous adverse effects, including cardiovascular and metabolic complications, loss of lean (muscle) mass, ${ }^{3}$ increase in fat mass and impaired physical performance, beyond that of natural ageing. ${ }^{4}$ Many ADT side effects may accelerate cognitive ageing, but evidence is inconclusive regarding effects to risk of developing cognitive impairment. ${ }^{5}$ However, heterogeneity in how cognitive impairment is defined and 
quantified has affected the degree to which cognitive impairment has been detected and reported. ${ }^{6}$

ADT has been negatively associated with cognitive performance in a range of domains, including reaction time, spatial and verbal memory, visuomotor speed and executive function, with the largest effects noted for visuomotor function. ${ }^{7}$ Nevertheless, it has been difficult to extricate the effects of PCa itself from those of ADT. Androgens, such as testosterone, are proposed to support male memory and visuospatial ability ${ }^{8}$ and may play a role in maintaining hippocampal plasticity, ${ }^{9}$ yet the differential contributions of cognitive and motor abilities to motor cognitive task performance are less clear. ${ }^{7}$ Declines in visuomotor performance associated with $\mathrm{ADT}$ use may be secondary to overall declines in motor function caused by ADT, rather than a direct effect of androgen depletion on cognitive processes. To our knowledge, no studies have evaluated the relationship between different cognitive domains reportedly affected by ADT and measures of muscle strength, cardiorespiratory fitness and physical function (mobility) in men with PCa. Importantly, the adverse effects of ADT on physical performance and cognition may be amenable to intervention, thereby reducing treatment-induced comorbidities from ADT.

The interdependence of physical and cognitive declines during normal ageing is speculated to be bidirectional. ${ }^{10}$ Age-related and disease-related increases in inflammation, changes in hormonal production and signalling (eg, insulin-like growth factor 1 (IGF-1) resistance, insulinreceptor substrate 1 and growth hormone dysregulation) and cardiometabolic dysfunction, can all directly contribute to both cognitive decline and muscle loss. ${ }^{11}$ There is also evidence that reduced muscle strength is prognostic for cognitive status in older adults, ${ }^{12}$ and declining muscle strength with age has been linked to an increased risk of Alzheimer's disease. ${ }^{13}$ Mobility impairments, particularly slow gait speed, have been associated with poorer cognitive function in older adults. ${ }^{12}$ The physiological mechanism(s) underlying this link between muscle strength, physical function and cognition are not completely clear. Skeletal muscle contractions upregulate various anti-inflammatory myokines, neurotrophic and growth factors that are important for neuroplasticity. ${ }^{14}$ Cardiorespiratory fitness may also protect against precursors to cognitive impairment such as cerebrovascular, endothelial and neurological degeneration. ${ }^{15}$ Associations between muscle strength and function with cognition have been identified in cancer survivors generally. ${ }^{16}$ However, whether reduced muscle strength, cardiorespiratory fitness and/or function are directly related to poorer cognitive function in men with PCa-receiving ADT is unknown.

Recent guidelines for measuring cognitive impairment in cancer research advocated the use of objective, validated, domain-specific measures and standardised criteria. ${ }^{6}{ }^{17}$ A variety of cognitive test batteries may be incorporated; however, prior studies have varied in their application and approach to analyses. ${ }^{18}$ Therefore, the primary aim of this study was to determine the strength and direction of any associations between muscle strength, cardiorespiratory fitness and physical function with cognitive function in men with PCa treated with and without ADT and non-PCa controls. A secondary aim was to compare the prevalence of cognitive impairment between men with PCa treated with and without ADT and non-PCa controls using standardised test batteries.

\section{MATERIALS AND METHODS}

This study was a nested cross-sectional study embedded alongside the baseline of a randomised controlled trial designed to evaluate the combined effects of exercise-training and nutritional supplementation on health outcomes in men with $\mathrm{PCa}$ treated with $\mathrm{ADT} .^{19}$ Recruitment of ADT-treated men was achieved via clinician referral from participating hospitals and support groups and newspaper advertisements from April 2014 to November 2017. PCON and CON were recruited via PCa support groups and newspaper advertisements from October 2014 to February 2016.

\section{Patient and public involvement}

Participants were not involved in the development of the research question, or design of the study. All participants were offered an individualised report outlining the results of their assessment in comparison to populationbased norms. Additionally, ADT-treated men were eligible to participate in a 12 month exercise and nutritional intervention. ${ }^{19}$

\section{Participants}

A total of 70 men aged with PCa pharmacologically treated with $\mathrm{ADT}$ for 12 weeks or greater (ADT), 52 men diagnosed with $\mathrm{PCa}$ not treated with ADT (prostate cancer control $[\mathrm{PCON}]$ ) and 70 men not diagnosed with PCa (control $[\mathrm{CON}]$ ) were included in the study. All men were aged 50-85 years. Exclusion criteria were: (1) non-English speaking; (2) any disorder known to affect bone, calcium or vitamin D metabolism (other than hypogonadism in the ADT group); (3) any current pharmacological intervention known to affect bone metabolism (other than ADT); (4) supplementation with protein, calcium $(>600 \mathrm{mg} /$ day) or vitamin D (>1000 IU/ day) in the past 3 months; (5) current progressive resistance training (more than one session/week) or weightbearing impact exercises $(>150 \mathrm{~min} /$ week $)$ in the past 3 months; (6) current smokers; (7) weight greater than $159 \mathrm{~kg}$; (8) plans to travel for longer than 6 weeks continuously within the following 52 weeks and (9) any absolute contraindications to exercise training (eg, musculoskeletal, cardiovascular or neurological disorders) according to the American College of Sports Medicine.

\section{Measures}

Standardised neurocognitive tests were used to assess cognitive function, including domains shown to be 
sensitive to disease and treatment-related changes in patients with cancer (eg, learning and memory, processing speed and executive function). ${ }^{6}$ This included the Trail Making Test to assess visuomotor speed (TMTA), task switching (TMTB) and executive function (TMTB minus TMTA).$^{20}$ Immediate recall, verbal learning and delayed memory were assessed using the Rey Auditory Verbal Learning Task (RAVLT, Rey auditory verbal learning test: trial 1, trial $1-5$ total, trial 7 respectively). ${ }^{21}$ The RAVLT is a word list measure for immediate and delayed recall, and verbal learning. ${ }^{21}$ Temporary verbal memory and verbal working memory were assessed with the DigitSpan test, which has also been validated in patients with cancer. ${ }^{22}$ The National Adult Reading Test was included to provide an estimate of cognitive reserve 6 , rather than as a measure proposed to be influenced by ADT.

The CogState Computerised Battery (CCB) (CogState, Melbourne, Australia) was also included to assess cognition, and has been customised and validated for patients with cancer. ${ }^{22}{ }^{23}$ Briefly, participants completed five tests that included game-like stimuli such as playing cards and tasks that measure a range of different cognitive domains: (1) Groton Maze Learning Test (GMT)—executive function, (2) detection task (DET)-processing speed and simple reaction time, (3) identification task (IDN) - choice reaction time and visual attention, (4) one card learning task (OCL) - attention and visual learning and (5) one back task (ONB) — working memory and visuomotor speed. ${ }^{23}$ Written and verbal instructions were given and a practice trial undertaken prior to each test. Outcomes were obtained for measures of reaction time of correct responses (in milliseconds) (IDN, DET, $\mathrm{ONB}$ ), proportion of correct responses (OCL) and total number of errors on five consecutive trials at a single session (GMT). The IDN, DET and ONB reaction time scores were $\log 10$ transformed, while the square root of the proportion of correct responses on the OCL task was arcsine transformed. ${ }^{24}$ These subtests were used to calculate raw scores, from which z-scores were derived using the mean and SD of the total sample. ${ }^{25}$ Three composite scores were calculated by averaging z-scores from specific tests: (1) global cognition (DET, IDN and OCL); (2) psychomotor-attention composite (DET and IDN), and (3) working-memory and learning composite (OCL and ONB) ${ }^{25}$ Higher composite scores represent better overall cognitive function.

We used two approaches to determine cognitive impairment (CI). Both approaches align with the International Cancer Cognition Task Force (ICCTF) proposed guidelines for assessing cognitive function in patients with cancer. ${ }^{26}$ The first method (standard battery $(\mathrm{SB})$ ) was based on scores 2-SD below the sample mean on at least two of eight tests from the TMT (TMTA, TMTB, TMTBTMTA), digit span and RAVLT tests (trials 1, 1-5, 7, DS forward, DS backwards). ${ }^{6} 17$ The second method used five cognitive domains from the CCB: psychomotor function/processing speed (detection), attention (identification), working memory (one-back), visual memory (one-card-learning) and executive function errors (the GMT). For this method, CI was indicated by a $\mathrm{z}$ score $-1.0 \mathrm{SD}$ or below the sample mean on three of the five tests. ${ }^{17}$ Both of these approaches are conservative selections within the range of appropriate test batteries possible under the ICCTF criteria, with the probability of exceeding cut-off criteria by chance $p \leq 0.015$ to $\leq 0.032$, respectively. ${ }^{27}$

As previously reported, ${ }^{19}$ lower limb muscle strength (kg) was measured using a three repetition maximum (3-RM) protocol (leg press, Synergy Omni Leg Press S-31OPD, Yatala, QLD, Australia) while upper limb strength was assessed using grip strength (Jamar Plus Digital, Lafayette Instrument Company, IN, USA), with the highest score $(\mathrm{kg})$ of three attempts from either the left or right hand recorded. Dual-task mobility was measured using the timed-up-and-go test with an additional cognitive task (DT TUG) using methods described previously. ${ }^{19}$ The time to complete the test was recorded with a stopwatch. Number of correct digits was also recorded; however, studies in non-cancer adults have indicated that an increase in cognitive load while dual tasking produces a trade-off effect to gait speed, regardless of the degree of difficulty of the cognitive task. ${ }^{28}$ Therefore, the DT TUG speed ( $\mathrm{sec}$ ) was used as an indication of physical performance (mobility) under cognitive load. Gait speed and estimated cardiorespiratory fitness were measured with the $4 \mathrm{~m}$ usual walk test and $400 \mathrm{~m}$ walk test, respectively. ${ }^{29}$ Resting blood pressure was calculated after an initial 10 min, seated rest using the means of the final two of three measurements taken by an automatic sphygmomanometer (TM-2655P, A\&D, Tokyo, Japan).$^{30}$ Height was measured using a stadiometer to the closest $0.1 \mathrm{~cm},(220$, Seca, Hamburg, Germany). Body mass was be measured using electronic scales (UC-321, A\&D, Tokyo, Japan) to the closest $0.1 \mathrm{~kg}$. Body mass index (BMI) was calculated to the closest $0.1 \mathrm{~kg} / \mathrm{m}^{2}$.

A questionnaire incorporating demographics, lifestyle and clinical information was used to obtain specific age, education, comorbidities, alcohol intake, PCa status and treatment details. Any discrepancies or queries with prior treatment details were resolved by checking medical records supplied by participants, or by their referring clinician. The Community Healthy Activities Model Programme for Seniors physical activity questionnaire was used to determine participation in a broad list of low, moderate and vigorous physical activities, with moderatevigorous physical activity (kJ per week) reported. ${ }^{31}$ The Depression Anxiety and Stress Scale was used to provide a measure of depression, anxiety, stress and general psychological distress, all of which have been shown to be altered by PCa diagnosis. ${ }^{32}$

\section{Data analysis}

All analyses were performed using Stata statistical software V.15 (StataCorp, College Station, Texas). Normality of the distribution of residuals was assessed visually via quantile-quantile plots and histograms. Between-group 
comparisons of participant characteristics were assessed by $\chi^{2}$ tests for categorical variables and analyses of variance for continuous variables. The strength and direction of associations between cognitive outcomes and measures of muscle strength, cardiorespiratory fitness and function were first assessed using Pearson's correlation coefficient in each group of men separately and all men combined. Analyses of covariance was then used to compare regression line slopes between groups for significant correlations between measures of muscle strength, cardiorespiratory fitness and function with cognitive function. To reduce the risk of type II error due to multiple comparisons, post hoc analyses applied the Tukey test. If no differences in slopes were identified, intercepts were compared between groups assuming identical slopes. ${ }^{33}$ An alpha level of $<0.05$ was adopted for all statistical tests.

\section{RESULTS}

Participant characteristics of each group are presented in table 1 . The mean (SD age of the total sample was 70 (7) years. Median (range) ADT duration was 12 (3-166) months. The treatment methods for ADT were as follows: goserelin $(n=40,57.1 \%)$, leuprorelin $(n=14,20 \%)$, goserelin and bicalutamide $(\mathrm{n}=5,7.1 \%)$, leuprorelin and bicalutamide $(\mathrm{n}=3,4.3 \%)$, triptorelin $(\mathrm{n}=3,4.3 \%)$, degarelix $(\mathrm{n}=2,2.9 \%)$, abiraterone $(\mathrm{n}=1,1.4 \%)$, degarelix and bicalutamide $(\mathrm{n}=1,1.4 \%)$ and enzalutamide $(\mathrm{n}=1,1.4 \%)$. Stage of PCa differed between ADT and PCON, with approximately two-thirds (64\%) of the former having had localised PCa (with or without previous prostatectomy) and most $(85 \%)$ of the latter unsure of PCa stage. On average, ADT had a higher BMI than PCON (mean difference, $\left.2.2 \mathrm{~kg} / \mathrm{m}^{2}, \mathrm{p}=0.010\right)$, but not CON. Men treated with ADT had 9.5\% ( $\mathrm{p}=0.003)$ and $13 \%(\mathrm{p}<0.001)$ lower grip strength compared with $\mathrm{PCON}$ and $\mathrm{CON}$, respectively. The $400 \mathrm{~m}$ walk test took $4.0 \%(\mathrm{p}=0.002)$ and $8.0 \%$ $(\mathrm{p}<0.001)$ longer for the ADT compared with PCON and CON, respectively. PCON participants had fewer anxiety symptoms than CON ( $p=0.007)$, but not ADT $(p=0.060)$. There was no significance between-group differences for alcohol consumption, total/cardiometabolic comorbidities, IQ, highest level of schooling or depressive/anxiety/ stress symptomatology.

Comparisons of cognitive function across the three groups are shown in table 2. On average, ADT had 17\% and $13 \%$ slower visuomotor speed (TMTA) than PCON $(\mathrm{p}=0.011)$ and CON $(\mathrm{p}=0.042)$, respectively. Both ADT and PCON had 0.5 SD $(\mathrm{p}=0.003)$ to $0.7 \mathrm{SD}(\mathrm{p}<0.001)$ slower simple reaction time (DET) and $0.4 \mathrm{SD}(\mathrm{p}=0.035)$ to $0.6 \mathrm{SD}(\mathrm{p}=0.001)$ slower choice reaction time when compared with CON. Choice reaction time (IDN) was also 0.4 SD to 0.6 SD slower in $\operatorname{ADT}(\mathrm{p}=0.035)$ and PCON ( $\mathrm{p}=0.001)$ compared with CON. Psychomotorattention composite scores (Cogstate) were lower in both ADT (-0.4 SD, p=0.006) and PCON (-0.6 SD, p<0.001) compared with CON. Finally, global cognition was lower in PCON $(-0.3 \mathrm{SD}, \mathrm{p}=0.019)$ with a trend for ADT $(-0.3$
$\mathrm{SD}, \mathrm{p}=0.073$ ), compared with CON. Notably, in further analyses that adjusted for obesity and anxiety, the only difference to the initial analyses was that working memory speed in ADT was slower compared with PCON $(\mathrm{p}=0.006)$, with no change to any other results.

Associations between muscle strength, cardiorespiratory fitness and physical function with cognition for each of the three groups separately are shown in table 3 . For leg press and grip strength, there were little or no associations with the RAVLT or digit span cognitive measures in any of the groups. In contrast, there were a number of significant correlations in each group between these two measures of muscle strength with the TMT and Cogstate composite cognitive outcomes (table 3), indicating that greater muscle strength was associated with better cognitive performance. However, when these associations were compared between groups, there were no group differences in the strength (slope) of the muscle strength to cognition relationships (online supplemental figure 1). Pooled data showed that when all men were combined, there were a number of significant associations between measures of lower and upper limb strength with several cognitive domains (online supplemental table 1)

The associations between physical function and cardiorespiratory fitness with cognition by group are shown in table 3. For gait speed, there were little or no associations with any of the cognitive measures in any of the groups. In contrast, there were a number of significant relationships within each of the groups between cardiorespiratory fitness and DT TUG with various cognitive measures. Specifically, the findings indicate that a greater level of fitness and dual task mobility were associated with better cognitive function across a number of domains for all. When these associations were compared between the three groups of men, the following significant differences in the slope were identified: (a) poorer cardiorespiratory fitness (slower $400 \mathrm{~m}$ walk time) was more strongly associated with a worse task switching performance (TMTB) in ADT compared with both PCON $(\mathrm{p}=0.018)$ and CON $(\mathrm{p}=0.032) \quad$ (figure 1A), (b) poorer cardiorespiratory fitness was more strongly associated with a worse executive function (TMTB-A) in ADT when compared with PCON ( $p=0.009)$ and CON ( $\mathrm{p}=0.037)$ (figure 1B), (c) slower dual task mobility (DT TUG) was more strongly associated with a worse psychomotor-attention composite scores in both ADT $(\mathrm{p}=0.027)$ and PCON $(\mathrm{p}=0.012)$ relative to CON (figure 2A), (d) slower dual task mobility was more strongly related to a worse working-memory and learning composite scores in ADT compared with CON ( $p=0.006)$, and to a lesser degree PCON $(p=0.051)$ (figure 2B), (e) slower dual task mobility was more strongly associated with a worse global cognition in both ADT $(\mathrm{p}=0.001)$ and PCON $(\mathrm{p}=0.031)$ relative to CON (figure 2C); (f) slower dual task mobility was similarly associated with poorer performance in tests of task switching (figure 3A) and executive function (figure 3B) for all groups. However, comparison of the intercepts revealed that for a given dual task mobility time, ADT performed 
Table 1 Characteristics of men with prostate cancer treated with androgen deprivation therapy (ADT), prostate cancer controls (PCON) and non-PCA controls (CON)

\begin{tabular}{|c|c|c|c|c|}
\hline Variable & ADT & PCON & CON & P-value \\
\hline $\mathrm{N}$ & 70 & 52 & 70 & \\
\hline Age, year & $71 \pm 6$ & $69 \pm 6$ & $69 \pm 7$ & 0.072 \\
\hline \multicolumn{5}{|l|}{ Stage of prostate cancer, $\mathrm{n}(\%)$} \\
\hline Localised/removed & $45(64.3)$ & $6(11.1)$ & - & $<0.001$ \\
\hline Advanced & $5(7.1)$ & $2(3.7)$ & - & \\
\hline Unknown & $20(28.6)$ & $44(84.6)$ & - & \\
\hline Previous prostatectomy, $\mathrm{n}(\%)^{*}$ & $34(48.6)$ & $36(69.2)$ & - & 0.022 \\
\hline Previous radiotherapy, $\mathrm{n}(\%)^{\star}$ & $48(68.6)$ & $12(23.1)$ & - & $<0.001$ \\
\hline Previous chemotherapy, $\mathrm{n}(\%)^{*}$ & $11(15.7)$ & $0(0.0)$ & - & 0.003 \\
\hline Active surveillance, $\mathrm{n}(\%)$ & - & $8(15.4)$ & - & - \\
\hline Weight, kg & $85.5 \pm 17.1$ & $82.4 \pm 13.5$ & $85.1 \pm 14.7$ & 0.073 \\
\hline Body mass index, $\mathrm{kg} / \mathrm{m}^{2}$ & $28.8 \pm 5.1$ & $26.6 \pm 4.0$ & $27.5 \pm 3.1$ & 0.013 \\
\hline Normal, n (\%) & $12(17.1)$ & $24(46.2)$ & $15(21.4)$ & 0.003 \\
\hline Overweight, n (\%) & 37 (52.9) & $19(36.5)$ & $41(58.6)$ & \\
\hline Obese, $n(\%)$ & $21(30.0)$ & $9(17.3)$ & $14(20.0)$ & \\
\hline Alcohol consumption, n (\%) & $32(53.3)$ & $16(32.7)$ & $32(45.7)$ & 0.095 \\
\hline If yes, $\mathrm{g} / \mathrm{d}$ & $25 \pm 20$ & $15 \pm 10$ & $23 \pm 19$ & 0.190 \\
\hline Total comorbidities, $\nmid n$ & $2 \pm 2$ & $2 \pm 2$ & $2 \pm 1$ & 0.449 \\
\hline Cardiometabolic comorbidities, $\neq n$ & $1 \pm 1$ & $1 \pm 1$ & $1 \pm 1$ & 0.354 \\
\hline Hypertension, n (\%) & $32(45.7)$ & $20(38.5)$ & $24(34.3)$ & 0.377 \\
\hline Heart disease, $\mathrm{n}(\%)$ & $20(28.6)$ & $7(13.5)$ & $20(28.6)$ & 0.096 \\
\hline Hypercholesterolaemia, n (\%) & $27(38.6)$ & $17(32.7)$ & $26(37.1)$ & 0.792 \\
\hline Type 2 diabetes, n (\%) & $7(10.0)$ & $5(9.6)$ & $4(5.7)$ & 0.608 \\
\hline IQ points & $117 \pm 5$ & $115 \pm 5$ & $116 \pm 5$ & 0.253 \\
\hline \multicolumn{5}{|l|}{ Highest level of schooling } \\
\hline Some high school, n (\%) & $5(7.1)$ & $3(5.8)$ & $8(11.4)$ & 0.198 \\
\hline Completed high school, n (\%) & $11(15.7)$ & $3(5.8)$ & $7(10.0)$ & \\
\hline Tech/trade certificate, n (\%) & $12(17.2)$ & $17(32.7)$ & $12(17.1)$ & \\
\hline Tertiary, n (\%) & $42(60.0)$ & $29(55.7)$ & $43(61.5)$ & \\
\hline Depressive symptoms, n (\%) & $10(16.1)$ & $9(17.3)$ & $14(20.0)$ & 0.838 \\
\hline Normal, n (\%) & 52 (83.9) & $43(82.7)$ & $56(80.0)$ & 0.602 \\
\hline Mild, n (\%) & $6(9.7)$ & $2(3.9)$ & $3(4.3)$ & \\
\hline Moderate, $\mathrm{n}(\%)$ & $2(3.2)$ & $5(9.6)$ & $6(8.6)$ & \\
\hline Severe, n (\%) & $0(0.0)$ & $1(1.9)$ & $2(2.8)$ & \\
\hline Extremely severe, n (\%) & $2(3.2)$ & $1(1.9)$ & $3(4.3)$ & \\
\hline Anxiety symptoms, n (\%) & $14(22.6)$ & $5(9.6)$ & $21(30.0)$ & 0.026 \\
\hline Normal, n (\%) & $48(77.4)$ & $47(90.4)$ & $49(70.0)$ & 0.205 \\
\hline Mild, n (\%) & $5(8.1)$ & $3(5.8)$ & $8(11.4)$ & \\
\hline Moderate, n (\%) & $5(8.1)$ & $0(0.0)$ & $5(7.2)$ & \\
\hline Severe, n (\%) & $3(4.8)$ & $2(3.8)$ & $4(5.7)$ & \\
\hline Extremely severe, n (\%) & $1(1.6)$ & $0(0.0)$ & $4(5.7)$ & \\
\hline Stress symptoms, n (\%) & $18(29.0)$ & $13(25.0)$ & $25(35.7)$ & 0.426 \\
\hline
\end{tabular}




\begin{tabular}{|c|c|c|c|c|}
\hline Variable & ADT & PCON & CON & P-value \\
\hline Normal, n (\%) & $44(71.0)$ & $39(75.0)$ & 45 (64.3) & 0.338 \\
\hline Mild, n (\%) & $3(4.8)$ & $5(9.6)$ & $9(12.9)$ & \\
\hline Moderate, $\mathrm{n}(\%)$ & $10(16.2)$ & $5(9.6)$ & $6(8.6)$ & \\
\hline Severe, n (\%) & $3(4.8)$ & $3(5.8)$ & $5(7.1)$ & \\
\hline Extremely severe, $\mathrm{n}(\%)$ & $2(3.2)$ & $0(0.0)$ & $5(7.1)$ & \\
\hline \multicolumn{5}{|l|}{ Muscle strength } \\
\hline Leg press, $\mathrm{kg}$ & $139.7 \pm 46.9$ & $143.5 \pm 42.0$ & $160.3 \pm 49.5$ & 0.048 \\
\hline Grip strength, kg & $37.9 \pm 6.5$ & $41.7 \pm 6.7$ & $43.3 \pm 8.0$ & $<0.001$ \\
\hline \multicolumn{5}{|l|}{ Physical function and fitness } \\
\hline Gait speed, m/sec & $1.42 \pm 0.21$ & $1.48 \pm 0.19$ & $1.50 \pm 0.22$ & 0.089 \\
\hline DT TUG, sec & $11.33 \pm 3.90$ & $11.64 \pm 4.58$ & $11.49 \pm 4.97$ & 0.933 \\
\hline $400 \mathrm{~m}$ walk, sec & $287.25 \pm 38.4$ & $275.92 \pm 32.0$ & $265.10 \pm 41.70$ & 0.004 \\
\hline
\end{tabular}

Data are mean \pm SD or number (percentage) within group. DT-TUG, dual task timed-up-and-go.

*ADT versus PCON only.

†Respiratory/pulmonary diseases, musculoskeletal, neurological, or immunological conditions.

‡Hypertension, type 2 diabetes, heart disease, hypercholesterolaemia.

more poorly on the task-switching test (TMTB intercept $\mathrm{p}=0.031$ ) and executive function test (TMTB-A intercept $\mathrm{p}=0.037$ ) compared with CON only. There were no other significant differences in slopes or intercepts between the groups (online supplemental figure 2).

Overall, nine (13\%) ADT men, two (3.9\%) PCON and seven $(10 \%)$ CON demonstrated cognitive impairment using the standard battery (figure $4 \mathrm{~A}$ ). In contrast, four $(5.7 \%)$ ADT men, five $(9.6 \%)$ PCON and four (5.7\%) CON had cognitive impairment using the CCB (figure 4B). The prevalence of cognitive impairment did not differ significantly between the three groups for the standard battery $(\mathrm{p}=0.234)$ or CCB $(\mathrm{p}=0.633)$.

\section{DISCUSSION}

The main finding from this study was that PCa, with and without ADT treatment, appears to alter the association between physical function and fitness (but not muscle strength), with various measures of cognitive function. For ADT-treated men specifically, stronger associations were evident between cardiorespiratory fitness and task switching ability and between cardiorespiratory fitness and executive function compared with both controls, indicating a stronger link between poorer fitness and reduced cognition in the ADT-treated men. Additionally, in both ADT and non-ADT men with PCa compared with $\mathrm{CON}$, there were stronger inverse associations between dual-task mobility and psychomotor attention as well as dual-task mobility and global cognition. However, the overall prevalence of cognitive impairment based on established criteria was relatively low and similar for men with PCa treated with and without ADT (4\%-13\%), and no different from controls $(6 \%-10 \%)$, although ADTtreated men had $17 \%$ and $13 \%$ lower visuomotor speed compared with PCON $(\mathrm{p}=0.011)$ and CON $(\mathrm{p}=0.042)$, respectively.

Previous research has shown that ADT use is associated with a deterioration in muscle strength, cardiorespiratory fitness and functional performance. ${ }^{34}$ In non-PCa lower muscle strength, fitness and function are linked to reduced cognitive function. ${ }^{34}$ Our study is the first to show that the relationship between some of these physical measures and cognitive function differs between ADT-treated men relative to PCa and/or CON. A causal association cannot be established between cardiorespiratory fitness and both task-switching ability and executive function. However, these outcomes offer insight into the previously reported mixed findings reported in prior research regarding the effects of ADT versus non-ADT on cognition in men with PCa. ${ }^{65}$ These adverse effects are supported by data showing that lower testosterone concentrations are associated with lower fitness and function $^{36}$ as well as deficits in memory ${ }^{37}$ and greater risk for Alzheimer's disease. ${ }^{38}$ It is possible that ADT-induced hypogonadism may accelerate age-related declines in brain processes, contributing in part to deteriorations in certain cognitive domains (eg, memory and visuospatial function). ${ }^{8}$ ADT-induced losses in cardiovascular fitness and function ${ }^{639}$ may also have secondary effects to cognition, creating a bidirectional and compound association.

An interesting finding from this study was that muscle strength was significantly lower in ADT-treated men compared with controls, but any associations between leg press muscle strength or grip strength with the various cognitive measures were either not significant or mixed across the three groups. This suggests that the ADT group had lower muscle strength, and while ageing may affect the association, ${ }^{10} \mathrm{PCa}$, with or without ADT, does 


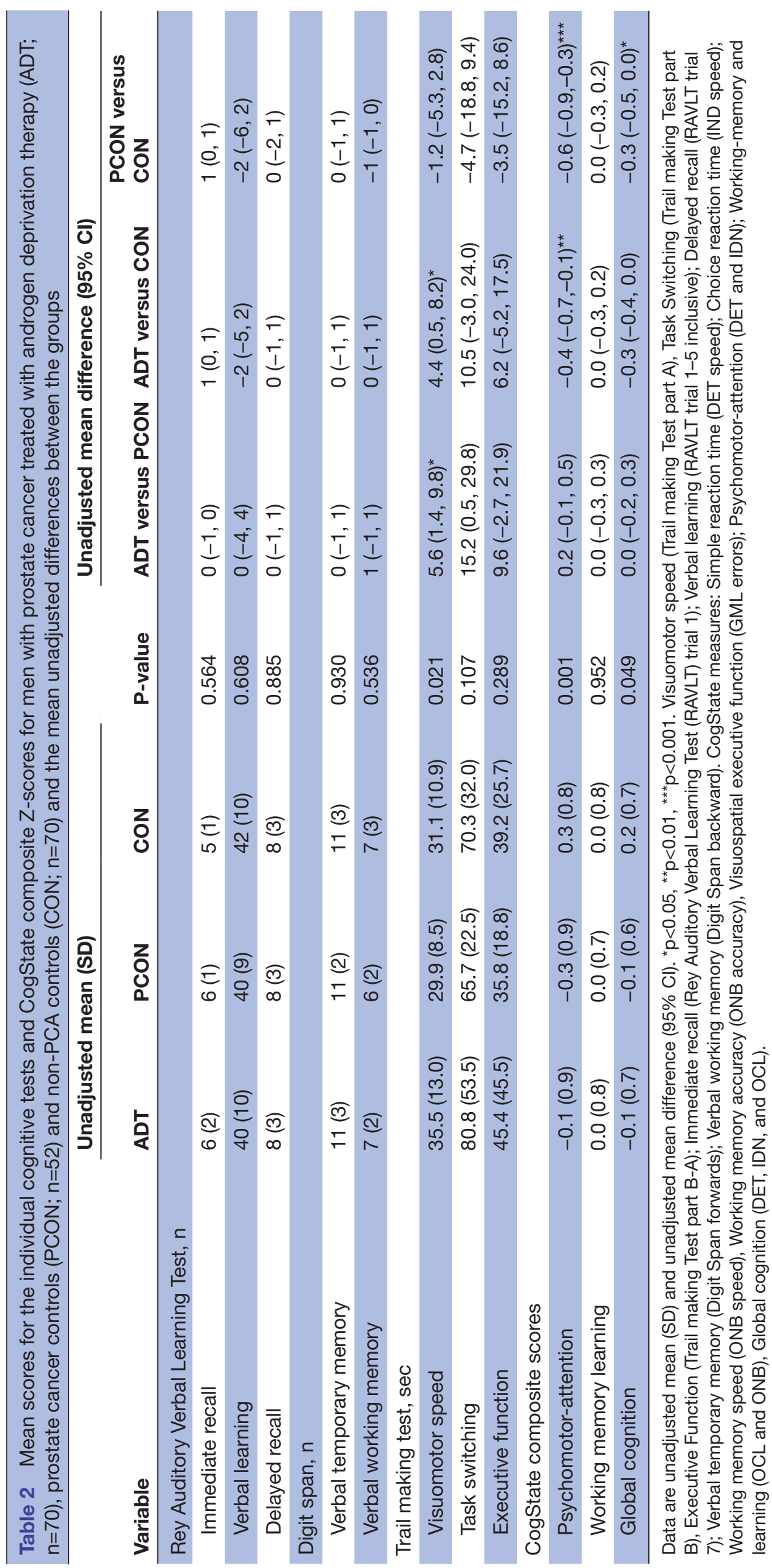


Table 3 The associations between muscle strength and function and different measures of cognition in men with prostate cancer treated with androgen deprivation therapy ( $A D T ; n=70)$, prostate cancer controls (PCON; $n=52$ ) and non-PCA controls (CON; $\mathrm{n}=70)$

\begin{tabular}{|c|c|c|c|c|c|c|}
\hline Variable & & Leg press 3RM & Grip strength & Gait speed & $400 \mathrm{~m}$ walk & TUGC \\
\hline Rey Auditory Verbal Lear & ig Test & & & & & \\
\hline Immediate recall & ADT & 0.24 & 0.083 & 0.144 & $-0.268^{\star}$ & -0.212 \\
\hline & PCON & 0.073 & -0.029 & 0.299 & $-0.354^{*}$ & $-0.428^{* *}$ \\
\hline & CON & 0.23 & 0.239 & 0.087 & $-0.346^{\star \star}$ & -0.232 \\
\hline Verbal learning & ADT & 0.071 & 0.077 & 0.122 & $-0.284^{*}$ & $-0.310^{*}$ \\
\hline & PCON & 0.169 & -0.032 & 0.261 & -0.287 & $-0.374^{*}$ \\
\hline & CON & 0.259 & $0.257^{*}$ & $0.191^{\star \star *}$ & $-0.458^{* *}$ & -0.247 \\
\hline Delayed recall & ADT & -0.013 & 0.054 & 0.076 & -0.245 & -0.247 \\
\hline & PCON & -0.099 & -0.097 & 0.173 & -0.266 & $-0.322^{*}$ \\
\hline & CON & 0.183 & 0.231 & 0.179 & $-0.359^{\star *}$ & -0.08 \\
\hline Digit span & & & & & & \\
\hline Temporary verbal memory & ADT & 0.169 & 0.199 & 0.157 & $-0.341^{*}$ & -0.241 \\
\hline & PCON & 0.087 & 0.111 & 0.021 & -0.029 & -0.253 \\
\hline & CON & 0.158 & 0.071 & 0.204 & $-0.334^{\star \star}$ & $-0.288^{\star}$ \\
\hline Verbal working memory & ADT & -0.051 & 0.038 & 0.097 & -0.028 & $-0.275^{\star}$ \\
\hline & PCON & $0.368^{*}$ & 0.148 & $0.309^{*}$ & -0.195 & $-0.349^{\star}$ \\
\hline & CON & 0.108 & 0.132 & 0.209 & -0.208 & -0.155 \\
\hline Trail making test & & & & & & \\
\hline Visuomotor speed & ADT & -0.179 & -0.172 & $-0.267^{*}$ & $0.372^{\star *}$ & $0.329^{*}$ \\
\hline & PCON & $-0.206^{\star}$ & -0.016 & -0.269 & $0.400^{* *}$ & $0.399^{\star *}$ \\
\hline & $\mathrm{CON}$ & $-0.446^{\star \star \star}$ & $-0.313^{\star *}$ & $-0.275^{*}$ & $0.282^{*}$ & 0.217 \\
\hline Task switching & ADT & -0.225 & $-0.259^{*}$ & -0.196 & $0.403^{\star *}$ & $0.347^{* \star}$ \\
\hline & PCON & $-0.357^{*}$ & -0.001 & -0.133 & 0.172 & $0.358^{*}$ \\
\hline & $\mathrm{CON}$ & $-0.483^{\star \star *}$ & $-0.262^{*}$ & -0.146 & $0.330^{* *}$ & 0.212 \\
\hline Executive function & ADT & -0.215 & -0.255 & -0.154 & $0.370^{* *}$ & $0.313^{*}$ \\
\hline & PCON & -0.321 & 0.006 & -0.038 & 0.026 & 0.249 \\
\hline & CON & $-0.416^{\star *}$ & -0.194 & -0.065 & $0.292^{*}$ & 0.172 \\
\hline CogState composite scol & & & & & & \\
\hline Psychomotor-attention & ADT & 0.261 & $0.307^{*}$ & 0.193 & -0.176 & $-0.389^{\star \star}$ \\
\hline & PCON & 0.069 & 0.213 & $0.287^{*}$ & -0.205 & $-0.473^{\star \star *}$ \\
\hline & CON & $0.358^{\star *}$ & $0.317^{\star *}$ & 0.1 & -0.235 & -0.073 \\
\hline Working memory learning & ADT & 0.264 & 0.126 & 0.146 & -0.144 & $-0.467^{\star * *}$ \\
\hline & PCON & 0.179 & -0.006 & 0.066 & -0.136 & -0.228 \\
\hline & $\mathrm{CON}$ & $0.303^{*}$ & $0.382^{\star *}$ & 0.132 & -0.237 & -0.098 \\
\hline Global cognition & ADT & $0.313^{*}$ & $0.254^{*}$ & 0.209 & $-0.289^{*}$ & $-0.522^{\star * *}$ \\
\hline & PCON & 0.174 & 0.162 & 0.248 & -0.23 & $-0.491^{\star * *}$ \\
\hline & $\mathrm{CON}$ & $0.402^{\star *}$ & $0.444^{\star * *}$ & 0.155 & $-0.294^{*}$ & -0.099 \\
\hline
\end{tabular}

Data are Pearson's correlation coefficient adjusted for multiple comparisons using Tukey's test. ${ }^{*} p<0.05,{ }^{* *} p<0.01,{ }^{* * *} p<0.001$ (bold). Visuomotor speed (Trail making Test part A), task switching (Trail making Test part B), executive function (Trail making Test part B-A); immediate recall (Rey Auditory Verbal Learning Test (RAVLT) trial 1); verbal learning (RAVLT trial 1-5 inclusive); delayed recall (RAVLT trial 7); verbal temporary memory (Digit Span forwards); verbal working memory (Digit Span backward). CogState measures: Simple reaction time (DET speed); choice reaction time (IND speed); working memory speed (ONB speed), working memory accuracy (ONB accuracy), visuospatial executive function (GML errors); psychomotor attention (DET and IDN); working memory and learning (OCL and ONB), global cognition (DET, IDN, and OCL). 


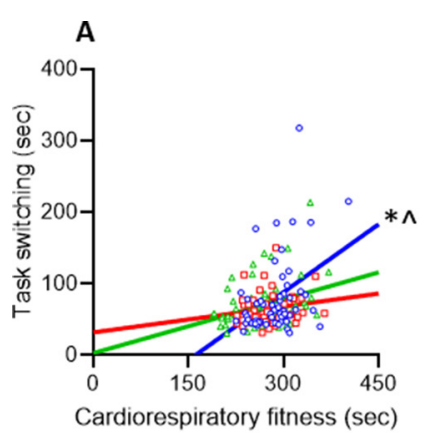

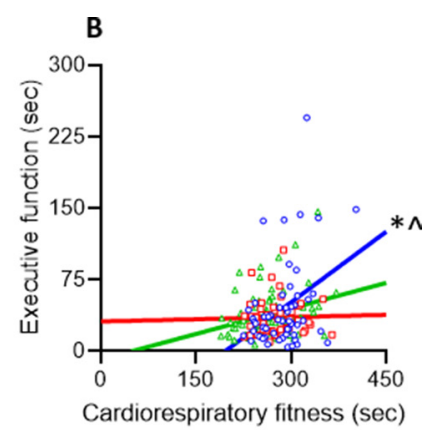

Cardiorespiratory fitness $(\mathrm{sec})$

Figure 1 Scatterplots of the relationship between cardiorespiratory fitness ( $400 \mathrm{~m}$ walk test performance) with task switching (trail making test $B ; A$ ) and executive function (trail making test $\mathrm{B}-\mathrm{A}$; $\mathrm{B}$ ) in men treated with androgen deprivation therapy (ADT), prostate cancer controls (PCON) and non-PCA controls (CON). Slope differences: * $\mathrm{p}<0.05$ compared with $\mathrm{PCON},{ }^{\wedge} \mathrm{p}<0.05$ compared with $\mathrm{CON}$.

not alter the degree to which strength impacts cognition. Mechanistically, muscle contractions can stimulate an increase in circulating brain-derived neurotrophic factor (BDNF), which is used for peripheral muscle metabolic processes. ${ }^{14}$ With regards to central cognitive processes, BDNF is thought to partially compensate for testosteronemediated plasticity in testosterone depletion. ${ }^{40}$ However, permeability of muscle-derived BDNF across the bloodbrain barrier is uncertain; ${ }^{14}$ therefore, peripheral muscle contractions are less likely to be relevant to cognition with or without ADT. By contrast, cardiorespiratory exercise is associated with increased circulating vascular and endothelial growth factors (eg, IGF-1), which may directly upregulate hippocampal BDNF, ${ }^{14}$ theoretically facilitating hippocampal synaptic plasticity in the absence of testosterone. Thus, those with greater strength may not necessarily benefit cognitively in the context of androgen depletion, but greater cardiorespiratory fitness may stimulate hippocampal BDNF and, thereby, partially compensate for a cognitive disadvantage with androgen

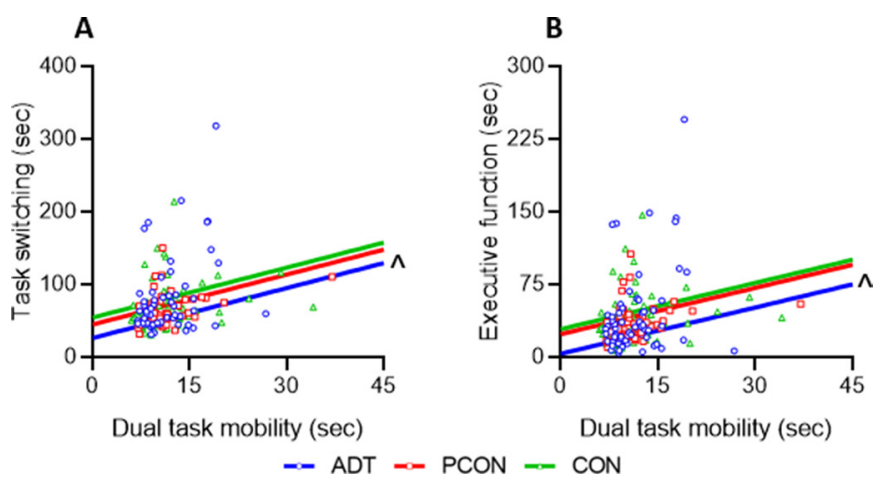

Figure 3 Scatterplot of the relationship between dual-task mobility (timed-up-and-go with cognitive task) with task switching (trail making test $B$; $A$ ) and executive function (trail making test $B-A$; $B$ ) in men treated with androgen deprivation therapy (ADT), prostate cancer controls (PCON) and non-PCA controls (CON). Since there were no differences in the slope, the regression lines assume identical slopes but there were between group differences in the intercepts: $1 p<0.05$ intercept difference compared with CON.

deficiency. As methods of measuring hippocampal BDNF in humans evolve, related future studies may consider, including such a measure.

Testosterone depletion has been linked with dementia risk and $\mathrm{AD}$, and prior research has suggested a role of $\mathrm{ADT}$ in progression to $\mathrm{AD}$ and dementia. ${ }^{26}$ It is worth noting that compromised cardiometabolic health increases the risk of neurocognitive impairment, and imaging studies have demonstrated that patients on ADT show reduced functional connectivity in brain structures (which may be vulnerable to vascular risk) associated with reaction time, attention, memory, information processing and spatial awareness. ${ }^{41} 42$ ADT promotes artherogenesis and is associated with compromised cardiovascular health. ${ }^{339}$ In the current study, matching samples based solely on age may have contributed to between-group differences in demographic characteristics; however; analysis of participant characteristics did not identify any between-group difference in habitual physical activity
A

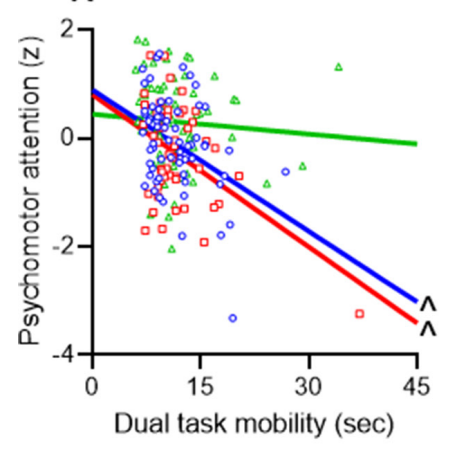

B

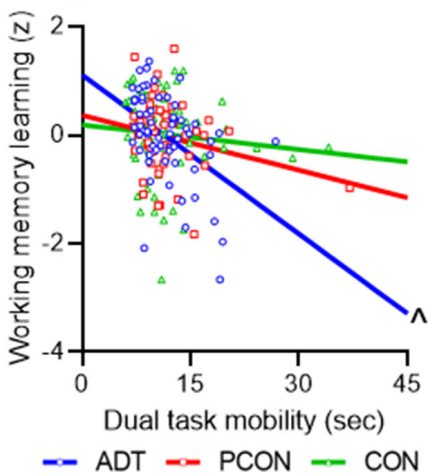

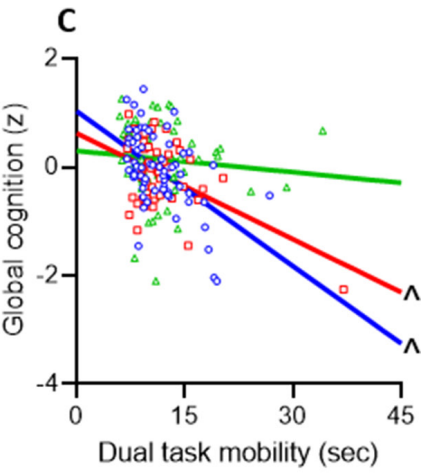

Figure 2 Scatterplots of the relationship between dual-task mobility (timed-up-and-go with cognitive task) with Cogstate psychomotor-attention composite score (A), Cogstate working memory-learning composite score (B) and Cogstate global cognitive function score (panel C) in men treated with androgen deprivation therapy (ADT), prostate cancer controls (PCON) and non-PCA controls (CON). Slope differences: ^ $p<0.05$ compared with CON. 
A

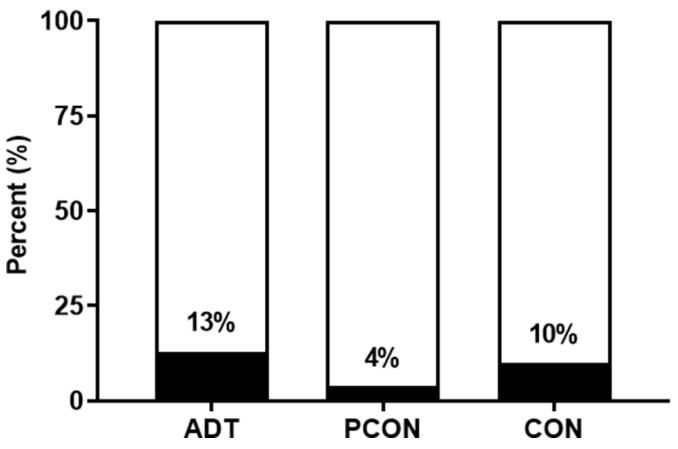

B

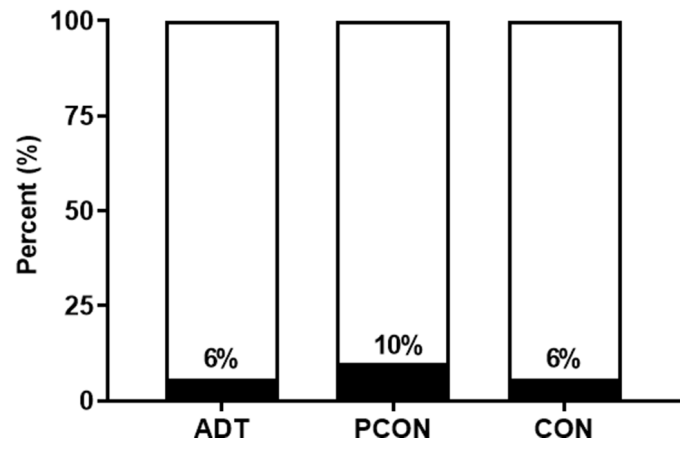

Figure 4 The prevalence of cognitive impairment in men treated with androgen deprivation therapy (ADT), prostate cancer controls (PCON) and non-PCA controls (CON) using the standard battery (A) consisting of the trail making test, Rey auditory verbal learning test and digit span, and the Cogstate computerised battery (B) consisting of the Groton maze learning test, detection task, identification task, one card learning task and one back task. $\square$-No cognitive impairment; $\square$-cognitive impairment.

levels or the number of comorbid conditions (including hypertension, type 2 diabetes, heart disease, hypercholesterolemia, respiratory/pulmonary diseases, musculoskeletal, neurological or immunological conditions). In addition, in an exploratory analysis when further adjustments were made to account for between-group differences in BMI and anxiety, results were unchanged. However, subclinical levels of cardiometabolic dysfunction have been identified in adults prior to clinical manifestation of disease. ${ }^{41}$ Changes to cardiometabolic health that have not reached clinically detectable levels (eg, subclinical atherosclerosis, arterial stiffness, endothelial dysfunction $)^{41}$ are less likely with increased cardiorespiratory fitness. ${ }^{39}$ These changes may be present in ADT men to a greater degree than for controls. Alternatively, the between-group differences in association between cardiorespiratory fitness and cognitive function may be due to the combined effects of testosterone suppression on neuroplasticity and cardiometabolic health, rather than a direct effect of ADT per se.

Another key finding from this study was the motorcognitive associations observed in both groups of men with $\mathrm{PCa}$, indicating that this relationship cannot be attributable solely to testosterone suppression. Furthermore, reaction time and psychomotor attention deficits were apparent in all men with PCa regardless of whether they received ADT. The dual-task mobility test comprised multiple motor components (lower limb power, initiation of stepping, acceleration, deceleration, turning ability) and has been linked with executive function and early detection of functional decline in older adults. ${ }^{43}$ This study did not measure serum hormones; however, elevated serum IGF-1 has been identified as a marker PCa risk, and serum IGF-1 suppression is a common target of cancer treatments. ${ }^{44}$ IGF-1 also plays an anabolic role in tissue regeneration and cerebral neuromodulation (facilitated by exercise), and age-related declines in serum IGF-1 may contribute to declines in cognitive and motor function for men with PCa. ${ }^{44}$ Thus, any cancer treatment that impacts IGF-1 may impact motor and cognitive function, which may explain an effect on cognition in PCa patients, ${ }^{45}$ thereby affecting both ADT and PCON to varying degrees. Future studies in men with PCa may consider the potential for endocrine and hormonal contributions to motor-cognitive associations for men with PCa. In addition, the merits of cardiorespiratory and multimodal exercise training in men with PCa are worth investigation, as these have shown benefits to function, cardiometabolic and cognitive health in older adults. ${ }^{46}$

This study did not identify any between-group differences in the prevalence of cognitive impairment using recommended criteria. Comparisons to prior studies are difficult as these studies have often yielded inconsistent findings, ${ }^{6}$ but an estimated $47 \%-69 \%$ of men with PCa treated with ADT have cognitive deficits in at least one domain over 6-12 months. ${ }^{46}$ These mixed findings are likely due to heterogeneous methodology, varying definitions of cognitive impairment and inconsistent use of objective, sensitive, validated tests (eg, an Auditory Verbal Learning Test, Digit Span and TMT). ${ }^{67}$ There are few studies using recommended criteria, ${ }^{18}$ and to our knowledge, none in ADT-treated men, which adjust for multiple comparisons in cognitive test batteries (as the number of cognitive tests used increases, the number of SD from the norm required for an abnormal classification should decrease proportionally). ${ }^{17}$ A prospective study observing ICCTF criteria (and adjusting for multiple comparisons) assessed objective cognitive performance in men with PCa $(n=58)$ with ADT and having had prostatectomy only. ${ }^{47}$ This study found that men receiving ADT for a period of 6 or 12 months were $70 \%$ and $50 \%$, respectively, more likely to demonstrate an impaired performance in executive function than controls, but based on the ICCTF criteria, cognitive impairment did not differ across groups. ${ }^{47}$ Our study also showed that the clinical utility of the two batteries used to determine cognitive impairment are comparable in this patient population. According to these criteria, there were no significant differences in cognitive impairment between ADT-treated men, PCa controls and CON in our study. It is possible that this 
finding is due to a recruitment bias inherent in the larger study. Men recruited for this study were offered a free 'health assessment', which may have led to an inclination for more health-focussed individuals to volunteer, biased participation towards individuals with healthy lifestyles and less chronic disease. ${ }^{48}$ In addition, batteries selected were highly specific (SB: $p \leq 0.015$, CCB: $p \leq 0.032) .{ }^{1727} \mathrm{We}$ also adjusted for multiple comparisons, therefore may have underestimated the prevalence of an abnormal result in any one test. Although our study did not identify a difference in the overall prevalence of cognitive impairment, across the specific cognitive domains, we identified deficits in visuomotor function (TMTA) in men treated with ADT compared with both controls. Consistent with our findings of a moderate effect of ADT on visuomotor function compared with non-ADT PCa $(\mathrm{g}=-0.50)$ and CON ( $\mathrm{g}=-0.36)$, a previous meta-analysis also reported a moderate effect size ${ }^{49}$ of ADT on visuomotor function $(\mathrm{g}=-0.67, \mathrm{p}=0.008) .{ }^{7}$ The visuomotor TMTA measure relies on integration of executive and fine-motor control of the upper limb and hand for visuomotor coordination. ${ }^{50}$ The absence of significant comparative deficits in other cognitive measures such as executive function (eg, TMTB-TMTA) in the ADT-treated men suggests that the impairment may be attributed to reduced motor capabilities rather than loss of executive control.

To our knowledge, this is the first study to assess the association between muscle strength, fitness and physical function with cognition in ADT-treated men compared with other patients with PCa not on ADT and healthy controls. A strength of this research is that it included a comprehensive battery of cognitive tests and a range of physical outcomes that related to strength, fitness and function. It also followed recent guidelines from the ICCTF with regards to defining cognitive impairment. ${ }^{6}$ Our study does contain limitations, which should be considered when interpreting results. First, this study was a nested cross-sectional substudy within a larger randomised controlled trial, including exercise training and nutritional supplements for ADT-treated men. ${ }^{19}$ Men on ADT recruited to the study had all been treated for a minimum of 3 months; however, adjusting for duration of treatment did not alter results. Second, there were differences in PCa stage between ADT and PCON, and a marked proportion (85\%) of PCON did not know the stage of their PCa. Finally, although causal direction cannot be established between fitness, physical function and certain cognitive domains with ADT, these findings support the maintenance of fitness and functional capacity for cognitive health in men with PCa.

In summary, reduced cardiorespiratory fitness, but not muscle strength, was associated with compromised cognitive function in men with PCa treated with ADT compared with controls. Additionally, dual-task mobility was associated with the psychomotor-attention composite and global cognition in men with PCa overall compared with controls. ADT-treated men showed significantly slower visuomotor speed compared with both controls; however, ADT did not alter prevalence of cognitive impairment overall. This is the first study to our knowledge that links compromised cardiorespiratory fitness with cognitive function in ADT-treated men, and poor dual-task mobility with cognitive function in men with PCa collectively. These findings reinforce the importance of maintaining physical fitness and functional capacity for cognitive health in men with PCa.

Correction notice This article has been corrected since it first published. Author names 'Robin N Daly' and 'Steve F Fraser' have been updated.

Acknowledgements The authors thank the study participants and referring clinicians involved in this study.

Contributors All authors have participated in the research and/or article preparation. Conception and design; NM, PJO, RD, SF; data collection; NM, PJO, JDV; analysis and interpretation of data; NM, PJO, RD, SF, HM; manuscript writing; all. Approval of final article: all authors have approved the final article. NM accepts full responsibility for the work and the conduct of the study, had access to the data, and controlled the decision to publish.

Funding The authors have not declared a specific grant for this research from any funding agency in the public, commercial or not-for-profit sectors.

Competing interests None declared.

Patient consent for publication Consent obtained directly from patient(s)

Ethics approval This study involves human participants and was approved by Ethics approval, which was granted by Deakin University Human Research Ethics Committee at Deakin University (HREC 2013-184), Peter MacCallum Cancer Centre (Project No:17/118) and Alfred Health (Project Number: 455/15). All participants gave their informed written consent prior to participation. Participants gave informed consent to participate in the study before taking part.

Provenance and peer review Not commissioned; externally peer reviewed. Data availability statement Data are available upon reasonable request.

Supplemental material This content has been supplied by the author(s). It has not been vetted by BMJ Publishing Group Limited (BMJ) and may not have been peer-reviewed. Any opinions or recommendations discussed are solely those of the author(s) and are not endorsed by BMJ. BMJ disclaims all liability and responsibility arising from any reliance placed on the content. Where the content includes any translated material, BMJ does not warrant the accuracy and reliability of the translations (including but not limited to local regulations, clinical guidelines, terminology, drug names and drug dosages), and is not responsible for any error and/or omissions arising from translation and adaptation or otherwise.

Open access This is an open access article distributed in accordance with the Creative Commons Attribution Non Commercial (CC BY-NC 4.0) license, which permits others to distribute, remix, adapt, build upon this work non-commercially, and license their derivative works on different terms, provided the original work is properly cited, appropriate credit is given, any changes made indicated, and the use is non-commercial. See: http://creativecommons.org/licenses/by-nc/4.0/.

\section{ORCID iDs}

Niamh L Mundell http://orcid.org/0000-0001-5406-3216

Patrick J Owen http://orcid.org/0000-0003-3924-9375

Jack Dalla Via http://orcid.org/0000-0002-1815-0838

Helen Macpherson http://orcid.org/0000-0002-3603-9359

Robin M Daly http://orcid.org/0000-0002-9897-1598

Steve F Fraser http://orcid.org/0000-0003-0202-9619

\section{REFERENCES}

1 Bray F, Ferlay J, Soerjomataram I, et al. Global cancer statistics 2018: GLOBOCAN estimates of incidence and mortality worldwide for 36 cancers in 185 countries. CA Cancer J Clin 2018;68:394-424.

2 Liede A, Hallett DC, Hope K, et al. International survey of androgen deprivation therapy (ADT) for non-metastatic prostate cancer in 19 countries. ESMO Open 2016;1:e000040.

3 Edmunds K, Tuffaha H, Galvão DA, et al. Incidence of the adverse effects of androgen deprivation therapy for prostate cancer: a systematic literature review. Support Care Cancer 2020;28:2079-93. 
4 Owen PJ, Daly RM, Livingston PM, et al. Lifestyle guidelines for managing adverse effects on bone health and body composition in men treated with androgen deprivation therapy for prostate cancer: an update. Prostate Cancer Prostatic Dis 2017;20:137-45.

5 Ryan C, Wefel JS, Morgans AK. A review of prostate cancer treatment impact on the CNS and cognitive function. Prostate Cancer Prostatic Dis 2020;23:207-19.

6 Wefel JS, Vardy J, Ahles T, et al. International cognition and cancer Task force recommendations to harmonise studies of cognitive function in patients with cancer. Lancet Oncol 2011;12:703-8.

7 McGinty HL, Phillips KM, Jim HSL, et al. Cognitive functioning in men receiving androgen deprivation therapy for prostate cancer: a systematic review and meta-analysis. Support Care Cancer 2014;22:2271-80.

8 Holland J, Bandelow S, Hogervorst E. Testosterone levels and cognition in elderly men: a review. Maturitas 2011;69:322-37.

9 MacLusky NJ, Hajszan T, Prange-Kiel J, et al. Androgen modulation of hippocampal synaptic plasticity. Neuroscience 2006;138:957-65.

10 Zammit AR, Robitaille A, Piccinin AM, et al. Associations between aging-related changes in grip strength and cognitive function in older adults: a systematic review. J Gerontol A Biol Sci Med Sci 2019;74:519-27.

11 Talbot $\mathrm{K}$, Wang $\mathrm{H}-\mathrm{Y}$, Kazi $\mathrm{H}$, et al. Demonstrated brain insulin resistance in Alzheimer's disease patients is associated with IGF-1 resistance, IRS-1 dysregulation, and cognitive decline. J Clin Invest 2012;122:1316-38

12 Chou M-Y, Nishita Y, Nakagawa T, et al. Role of gait speed and grip strength in predicting 10-year cognitive decline among communitydwelling older people. BMC Geriatr 2019;19:186.

13 Buchman AS, Wilson RS, Boyle PA, et al. Grip strength and the risk of incident Alzheimer's disease. Neuroepidemiology 2007;29:66-73.

14 Delezie J, Handschin C. Endocrine crosstalk between skeletal muscle and the brain. Front Neurol 2018;9:698.

15 Kennedy G, Hardman RJ, Macpherson H, et al. How does exercise reduce the rate of age-associated cognitive decline? A review of potential mechanisms. J Alzheimers Dis 2017:55:1-18.

16 Yang L, Koyanagi A, Smith L, et al. Hand grip strength and cognitive function among elderly cancer survivors. PLOS One 2018;13:e0197909.

17 Ingraham LJ, Aiken CB. An empirical approach to determining criteria for abnormality in test batteries with multiple measures. Neuropsychology 1996;10:120-4.

18 Sun M, Cole AP, Hanna N, et al. Cognitive impairment in men with prostate cancer treated with androgen deprivation therapy: a systematic review and meta-analysis. J Urol 2018;199:1417-25.

19 Owen PJ, Daly RM, Livingston PM, et al. Efficacy of a multicomponent exercise programme and nutritional supplementation on musculoskeletal health in men treated with androgen deprivation therapy for prostate cancer (impact): study protocol of a randomised controlled trial. Trials 2017;18:451.

20 Kayl AE, Collins R, Wefel JS, et al. Neuropsychological assessment of adults with cancer. In: Meyers CA, Perry JR, eds. Cognition and cancer. Cambridge: Cambridge University Press, 2008: 44-55.

21 Schmidt M. Rey auditory verbal learning test. 625 Alaska Avenue, Torrance, CA Western Psychological Services (WPS), 1996.

22 Sjøgren P. Psychomotor and cognitive functioning in cancer patients. Acta Anaesthesiol Scand 1997;41:159-61.

23 CogState. CogState research manual Copyright @ (In., 6 EDN: CogState, LTD, 2011.

24 Lim YY, Ellis KA, Harrington K, et al. Use of the CogState brief battery in the assessment of Alzheimer's disease related cognitive impairment in the Australian imaging, biomarkers and lifestyle (AIBL) study. J Clin Exp Neuropsychol 2012;34:345-58.

25 CogState. Research guidelines for data analysis August, 2017, 2017: 1-15.

26 Tae BS, Jeon BJ, Shin SH, et al. Correlation of androgen deprivation therapy with cognitive dysfunction in patients with prostate cancer: a nationwide population-based study using the National health insurance service database. Cancer Res Treat 2019;51:593-602.

27 Vucic K, Jelicic Kadic A, Puljak L. Survey of Cochrane protocols found methods for data extraction from figures not mentioned or unclear. J Clin Epidemiol 2015;68:1161-4.
28 Maclean LM, Brown LJE, Khadra $\mathrm{H}$, et al. Observing prioritization effects on cognition and gait: the effect of increased cognitive load on cognitively healthy older adults' Dual-task performance. Gait Posture 2017;53:139-44.

29 Simonsick EM, Fan E, Fleg JL. Estimating cardiorespiratory fitness in well-functioning older adults: treadmill validation of the long distance corridor walk. J Am Geriatr Soc 2006;54:127-32.

30 Kobalava ZD, Kotovskaya YV, Babaeva LA, et al. Validation of TM2655 oscillometric device for blood pressure measurement. Blood Press Monit 2006;11:87-90.

31 Stewart AL, Mills KM, King AC, et al. Champs physical activity questionnaire for older adults: outcomes for interventions. Med Sci Sports Exerc 2001;33:1126-41.

32 Bill-Axelson A, Garmo H, Lambe M, et al. Suicide risk in men with prostate-specific Antigen-Detected early prostate cancer: a nationwide population-based cohort study from PCBaSe Sweden, 2010.

33 Zar JH. Biostatistical analysis. Second edition. edn. Prentice-Hall, 1984.

34 Voelcker-Rehage C, Godde B, Staudinger UM. Physical and motor fitness are both related to cognition in old age. Eur $\mathrm{J} \mathrm{Neurosci}$ 2010;31:167-76.

35 Treanor CJ, Li J, Donnelly M. Cognitive impairment among prostate cancer patients: an overview of reviews. Eur J Cancer Care 2017;26:e12642.

36 Orwoll E, Lambert LC, Marshall LM, et al. Endogenous testosterone levels, physical performance, and fall risk in older men. Arch Intern Med 2006;166:2124-31.

37 Moffat SD, Zonderman AB, Metter EJ, et al. Longitudinal assessment of serum free testosterone concentration predicts memory performance and cognitive status in elderly men. J Clin Endocrinol Metab 2002;87:5001-7.

38 Pintana H, Chattipakorn N, Chattipakorn S. Testosterone deficiency, insulin-resistant obesity and cognitive function. Metab Brain Dis 2015;30:853-76.

39 Wall BA, Galvão DA, Fatehee N, et al. Reduced cardiovascular capacity and resting metabolic rate in men with prostate cancer undergoing androgen deprivation: a comprehensive cross-sectional investigation. Adv Urol 2015;2015:976235.

40 Skucas VA, Duffy AM, Harte-Hargrove LC, et al. Testosterone depletion in adult male rats increases mossy fiber transmission, LTP, and sprouting in area CA3 of hippocampus. J Neurosci 2013;33:2338-55.

41 Waldstein SR, Wendell CR. Neurocognitive function and cardiovascular disease. J Alzheimers Dis 2010;20:833-42.

$42 \mathrm{Chao} \mathrm{HH}$, Hu S, Ide JS, et al. Effects of androgen deprivation on cerebral morphometry in prostate cancer patients - an exploratory study. PLoS One 2013;8:e72032-6.

43 Herman T, Giladi N, Hausdorff JM. Properties of the 'timed up and go' test: more than meets the eye. Gerontology 2011;57:203-10.

44 Devin JL, Bolam KA, Jenkins DG, et al. The influence of exercise on the insulin-like growth factor axis in oncology: physiological basis, current, and future perspectives. Cancer Epidemiol Biomarkers Prev 2016;25:239-49.

45 Jarzemski P, Brzoszczyk B, Popiołek A, et al. Cognitive function, depression, and anxiety in patients undergoing radical prostatectomy with and without adjuvant treatment]]\&gt 2019;15:819-29.

46 Mundell NL, Daly RM, Macpherson $\mathrm{H}$, et al. Cognitive decline in prostate cancer patients undergoing ADT: a potential role for exercise training. Endocr Relat Cancer 2017;24:R145-55.

47 Gonzalez BD, Jim HSL, Booth-Jones M, et al. Course and predictors of cognitive function in patients with prostate cancer receiving androgen-deprivation therapy: a controlled comparison. $J$ Clin Oncol 2015;33:2021-7.

48 Harris TJ, Victor CR, Carey IM, et al. Less healthy, but more active: opposing selection biases when recruiting older people to a physical activity study through primary care. BMC Public Health 2008;8:182.

49 Hedges LV. Distribution Theory for Glass's Estimator of Effect size and Related Estimators. Journal of Educational Statistics 1981;6:107-28.

50 Salthouse TA. What cognitive abilities are involved in trail-making performance? Intelligence 2011;39:222-32. 